\title{
Ileo-Ileal Knot Causing Intestinal Obstruction A Case Report
}

\author{
${ }^{1}$ Dr.LokeshwariKalaichelvan, ${ }^{2}$ Dr.Srinivasan Ulagendra Perumal, ${ }^{3}$ Dr. Kannan \\ Ross, ${ }^{4}$ Dr.Balakrishnan Subramanian, \\ ${ }^{I}$ Department Of General Surgery, Government Royapettah Hospital, Chennai, India. \\ ${ }^{2}$ Department Of Surgical Gastroenterology, Government Royapettah Hospital, Chennai, India \\ ${ }^{3}$ Department Of General Surgery, Government Royapettah Hospital, Chennai, India \\ ${ }^{4}$ Department Of General Surgery, Government Royapettah Hospital, Chennai , India
}

\begin{abstract}
Intestinal knot formation is a rare cause of closed loop obstruction. It was first described by Riverius in the 16th century. The lesion consists of a knot formed between two loops of bowel, causing mechanical obstruction. Here we present a case of acute intestinal obstruction due to ileo-ileal knotting, which was surgically managed by emergency laparotomy. The ileo-ileal knot is a very rare surgical emergency that can rapidly evolve to gangrene of the affected bowel segment. It is very difficult to diagnose this condition preoperatively. High index of suspicion and early surgical intervention greatly reduces morbidity and mortality. Keywords:ileoileal knot, intestinal knot, intestinal obstruction
\end{abstract}

\section{Introduction}

Intestinal knot formation was first described by Riverius in the $16^{\text {th }}$ century and by Rokitansky in 1836. The lesion consists of either of an intertwining or a knot formed between two loops of bowel, causing a mechanical intestinal obstruction and leading to strangulation of one or both loops. Three types of intestinal knotting are described- ileo-ileal, ileo-sigmoid and knot formed between bowel and Meckel's diverticulum or appendix. Here we present a case of ileo-ileal knotting which is a rare cause of intestinal obstruction.

\section{Case presentation}

A 65 year old male presented with diffuse abdominal pain associated with nausea and vomiting for two days. He had constipation and abdominal distension for the past three days with a history of fever at the onset of abdominal pain. He is a known hypertensive on regular treatment for three years with no other co-morbid illness. There was no history of previous abdominal surgery. On examination, he had tachycardia, normal blood pressure and a moderately distended abdomen with diffuse tenderness. Bowel sounds were absent and digital rectal examination revealed an empty rectum. Plain X-ray abdomen- erect showed dilated bowel loops (Fig: 1). Computed tomography (CT) showed dilated small bowel loops with collapsed large bowel, suggesting a small bowel obstruction (Fig:2, Fig:3). He was taken up for emergency laparotomy. During laparotomy, about $200 \mathrm{ml}$ of toxic fluid along with knotting of a loop of ileum was found. Further examination revealed a volvulus of distal part of ileum with congestion of the bowel (Fig:4, Fig:5). Ileal knot released; volvulus de-rotated (Fig: 6) and a warm compression was given to the bowel. The bowel loop turned pink and regained peristalsis. Thorough peritoneal wash was given and abdomen closed with a tube drain. Oral feeding started on the fifth postoperative day was tolerated well by the patient, who had a good postoperative recovery and was discharged on the eighth postoperative day.

\section{Discussion}

The initial mention of intestinal knotting was by Riverius in 16th century while a more detailed description of this rare entity was made in 1836 by Rokitansky. The lesion consists of either of an intertwining or a knot, formed between two loops of bowel, causing a complex intestinal obstruction. These are also known as compound volvulus ${ }^{[1]}$ Three types of intestinal knots are distinguished: Ileoileal; ileosigmoid and knots formed between the bowel and Meckel's Diverticulum or the appendix ${ }^{[2]}$. The latter are not considered as intestinal knots by some authors because adhesions play an important and consistent role in their formation ${ }^{[3]}$. Once the knot is formed it leads to obstruction, ischaemia and gangrene of the involved bowel rapidly. It is unusual in younger age group, most commonly presenting in fourth decade. Due to its rarity its sex predilection cannot be identified. The predisposing factors of these entities may be the coexistence of an elongated, mobile, small bowel mesentery ${ }^{[4]}$ and the redundant sigmoid $\operatorname{colol}^{[5]}$ with a narrow base, lack of mesenteric fat, ingestion of high bulk diet after fasting ${ }^{[6]}$. Most cases of ileal knot reported in Ibadan during Ramadan are believed to be due to narrow mesentery with high volume intake after fasting. ${ }^{[7]}$ It is very difficult to diagnose this condition preoperatively, as the patient presents with acute abdomen with a broad differential diagnosis and diagnosis is often made intraoperatively. It is a type of closed loop obstruction. In ileo-ileal knotting one part of 
ileum remains static and the other part usually twines round the static part. When all segments are viable untying the knot may be enough since recurrence is uncommon. When irreversible ischemia is present enterotomy decompression should be done first and then resection of the nonviable part of bowel is proceeded with. Manipulation of the knot with intention of untying is not recommended in ischaemic bowel, because of a high risk of perforation ${ }^{[8]}$. After resection, a primary end to end anastomosis of the small bowel should be done if the distal ileum is not affected. If the distal segment is closer than $10 \mathrm{~cm}$ to the ileocecal valve, end to side ileocolic anastomosis is preferred.

\section{Figures}

Figure 1: Xray abdomen showing dilated bowel loops

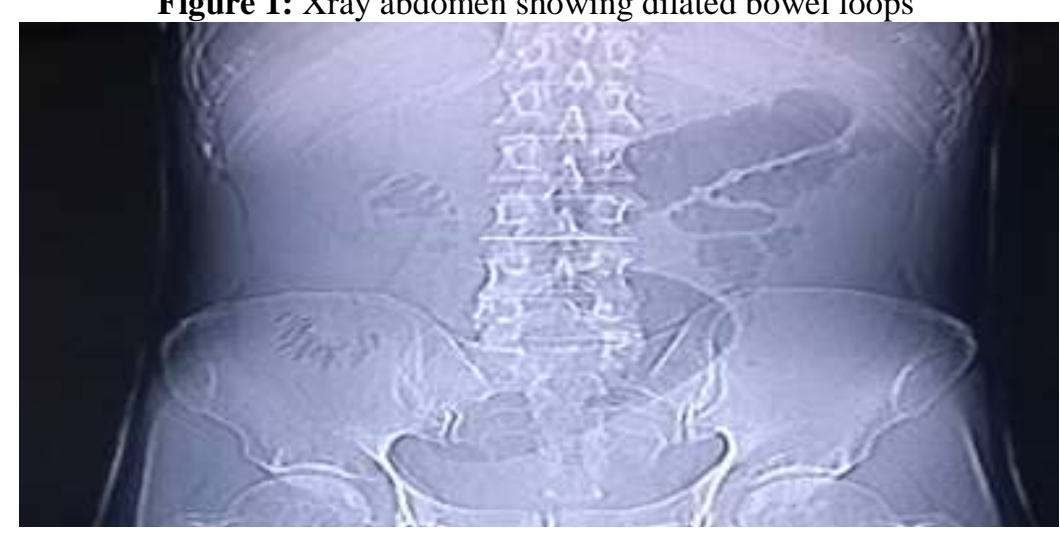

Figure2: Computed Tomography Abdomen Showing Dilated Small Bowel Loop

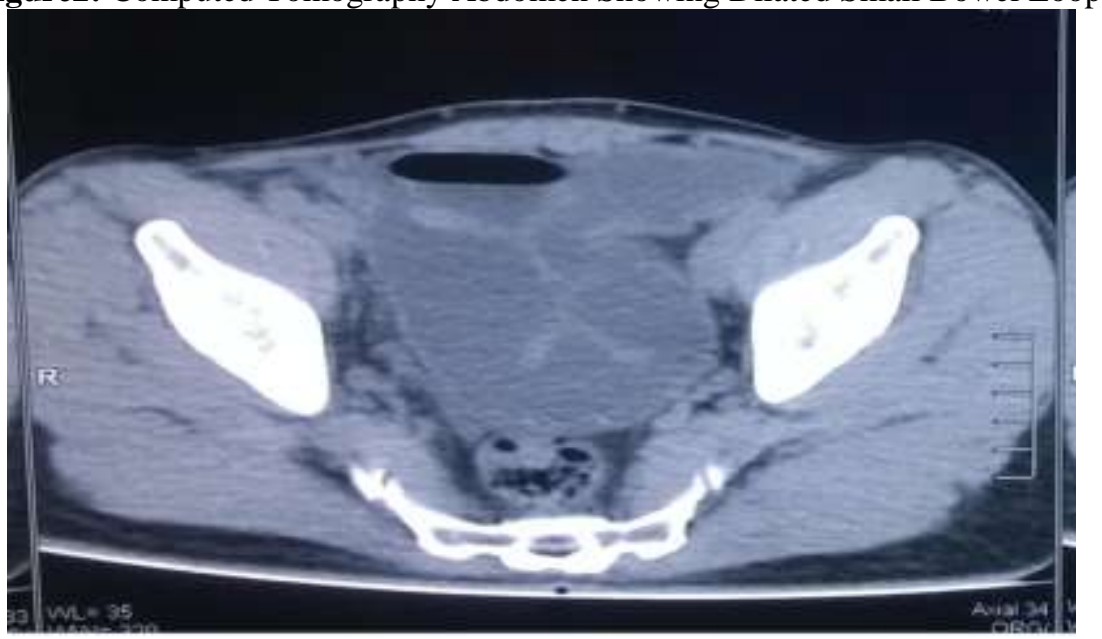

Figure 3: CT Abdomen Showing Dilated Fluid Filled Small Bowel Loop

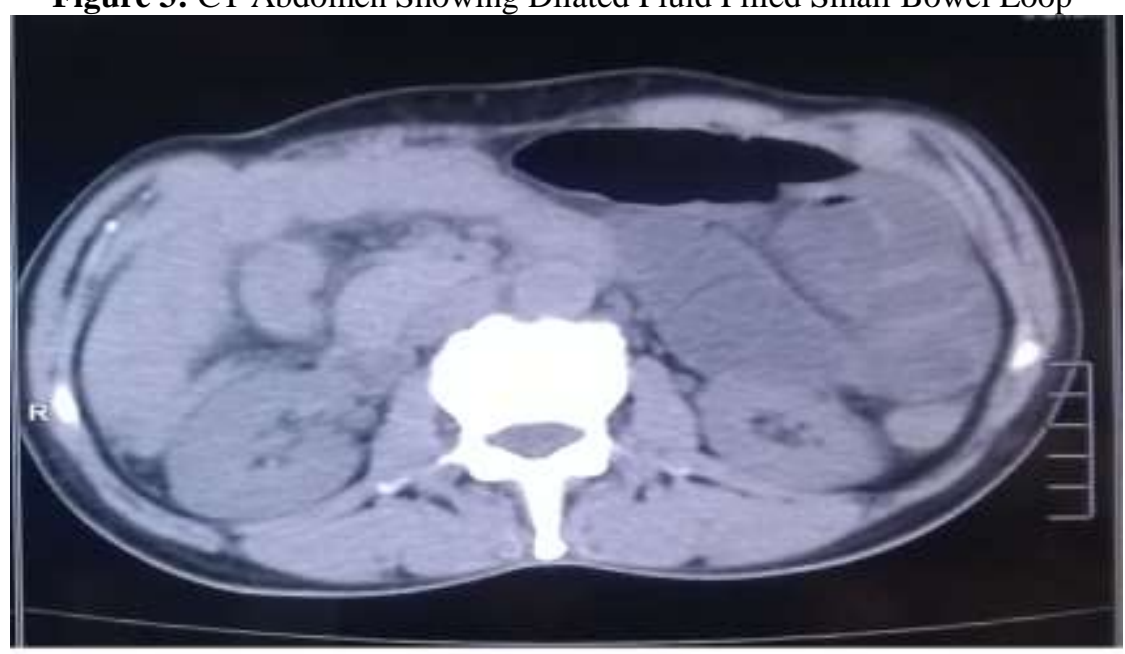

Figure 4: Intraoperative Photograph Showing Ileoileal Knotting 


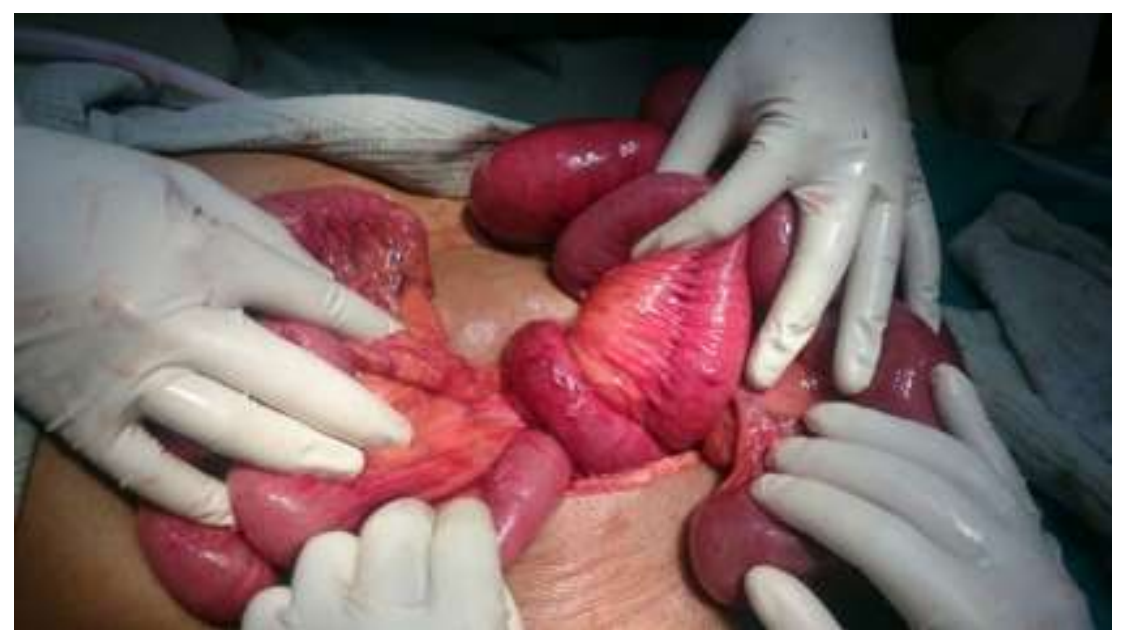

Figure5: Intraoperative Photograph Showing Ileoileal Knotting

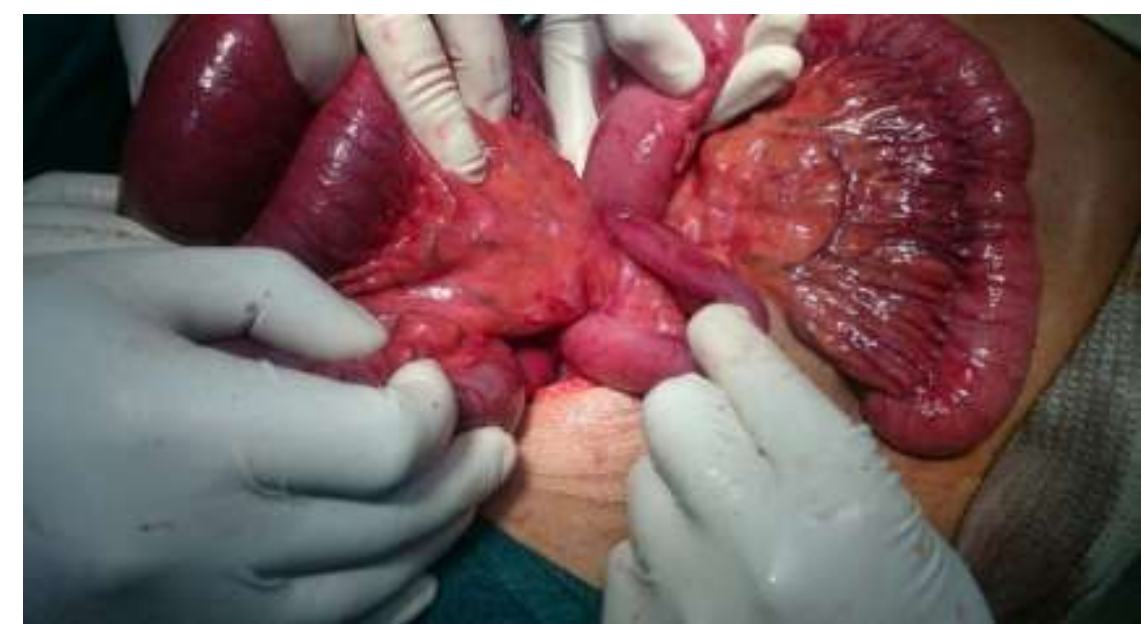

Figure 6: After Untwisting The Knot

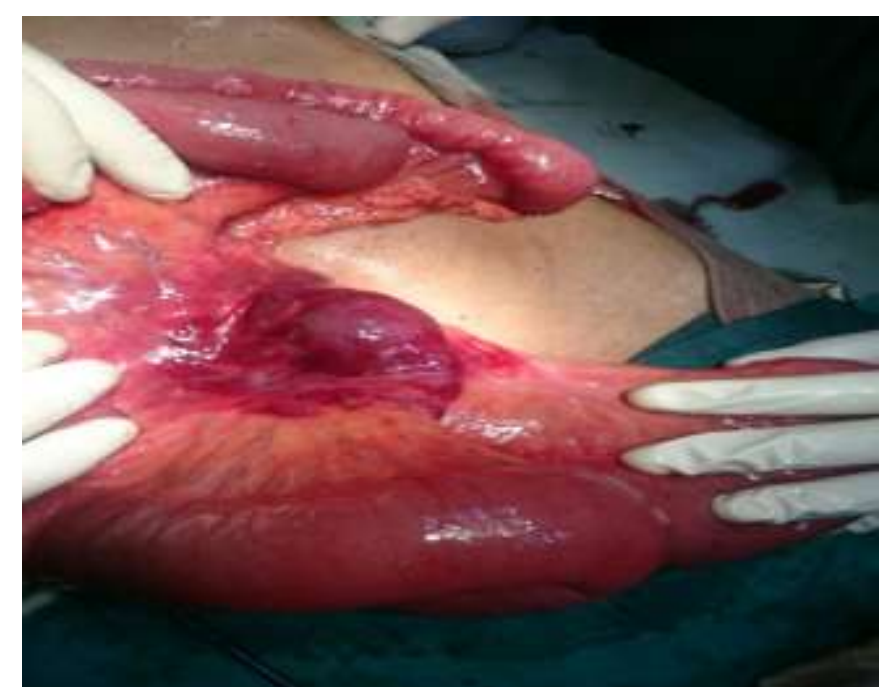

IV. Conclusion

Due to its rarity and vague presentation, a high degree of suspicion with early surgical intervention greatly reduces mortality and morbidity of this rapidly progressive fatal condition.

\section{References}

[1]. Kallio.E.D. 1932 . Die Knotenbildungen des Darmes. Acta chirurgica Scandinavia suppl,21,1-276

[2]. Mikal S,Byers JA.,Closed loop obstruction of the ileum due to an appendiceal knot J Am Med Assoc 1956;160;49-50 . 
[3]. Vaez-Zadeh. K M.D., Dutz,W. M.D., Ileosigmoid knotting, Annals of surgery, Volume172, number 6, December 1970.

[4]. Shepherd JJ. The epidemiology and clinical presentation of sigmoid volvulus .Br J Surg 11969;56;353 -359 .

[5]. Nikolaos Andromanakos et al. An unusual synchronous ileosigmoid and ileoileal knotting: a case report, journal of medical case reports , june 2014

[6]. Shackelfords surgery of the alimentary tract, Charles J. Yeo, Elsevier Saunders, $7^{\text {th }}$ edition pg $875-75$

[7]. Maingots abdominal operations, Michael j Zinner, Stanley W Ashley, McGrawHill $12^{\text {th }}$ edition pg587

[8]. Uday SK, Venkata PKCH, Kumar S. Ileo-ileal knot causing small bowel gangrene: an unusual presentation. International Journal of case reports and Images 2012;3(5):28-30 